\title{
Blended Learning, una alternativa para E-learning: Un modelo Educativo
}

\section{Blended Learning, an alternative to E-learning: an educational model}

Jaime Polania Perdomo

Magister en Tecnologías. Universidad Surcolombiana. Neiva. Colombia. jpolania@usco.edu.co

\section{Resumen}

La utilización de las tecnologías de la información y la comunicación (TICs) en el ámbito de la educación superior plantea cambios significativos y nuevas opciones en la búsqueda de mejorar el proceso de enseñanzaaprendizaje, sin embargo, la formación totalmente a distancia utilizando TICs o la enseñanza tradicional (cara a cara), poseen fortalezas y debilida-des propias que han conducido al surgimiento de nuevas modalidades de metodología educativa (Singh \& Reed, 2001).

Una modalidad de aprendizaje moderno es la metodología BlendedLearning (proceso de enseñanza que combina la formación presencial, con la formación virtual), que surge como una alternativa para e-Learning, debido a los inconvenientes encontrados por los estudiantes que seguían cursos de formación y autoformación exclusivamente virtuales; entre ellos, los altos niveles de deserción entre estudiantes y el aislamiento en ambientes de formación puramente virtuales, demuestran que el proceso de enseñanza-aprendizaje presencial no son reproducidas con la misma intensidad y calidad con las nuevas tecnolo-gías.

Palabras claves: E-learning, m-learning, Blended Learning, TICs, educación a distancia, educación virtual, asincrónico, autoprogramación, chat, blogs, news, wikis.

\section{Abstract}

The use of information and communication technology in higher education poses significant changes and new options in the effort to improve the teaching-learning process. However, distance learning using information and communication technology and traditional learning methods (face to face) both have their own strengths and weaknesses which have led to the emergence of new educational methodologies (Singh \& Reed, 2001).

A modern learning method is the Blended Learning method (a learning process which combines classroom-based learning, with virtual learning) which has emerged as an alternative to e-Learning, due to the difficulties found by students who follow completely virtual vocational and self-training courses. For example, high drop-out rates among students and the isolation that comes with purely virtual learning environments demonstrate that new technology cannot reproduce the same intensity and quality of the face-to-face learning process.

Keywords: E-learning, m-learning, Blended Learning, TICs, distance learning, virtual learning, asynchronous, self-scheduling, chat, blogs, news, wikis

\section{Educación a distancia}

La educación a distancia no es reciente, es una modalidad educativa que surge como una respuesta a la necesidad de educación permanente, a la demanda social de educación para que esta llegue a todos los sectores de la sociedad considerando regiones apartadas y de difícil acceso. Según Vásquez (2007), es una opción para las personas adultas debido a que cuenta con grandes bondades, y hace accesible la formación continua de las personas, pero es indispensable hacer mención que dicha modalidad también cuenta con su grado de dificultad u obstáculos para los estudiantes.

La educación a distancia ha sido definida de diversas formas por varios autores. Autores como Serrano 
(1999), propone que se trata de una modalidad educativa que comprende una situación formal de enseñanza y aprendizaje donde el que enseña se encuentra en una dimensión temporoespacial diferente del que aprende, por lo que establecen una relación a través de diferen-tes medios y modelo de educación, para facilitar la transmisión del conocimiento con posibilidad de dialogo e interacción síncrona 0 asíncrona separado.

También es considerada una vía para lograr la incorporación al sistema educativo de diferentes segmentos de una población con dificultades para cumplir con los requisitos de asistencia propios de los cursos regulares, y mantenerlos en el mismo, en los diferentes niveles de formación, y particularmente en el nivel universitaria, que conduzca a la obtención de una certificación como en la educación continuada, (Jiminián, 2006).

Aunque la educación a distancia ha sido una opción desde el siglo XIX, es hasta mediados del siglo XX que las instituciones educativas incursionan en investigaciones para dar cuenta de este modelo educativo e ir dando bases para modelo teóricos que lo sustenten (Gil, 2000). Cuatro elementos han sido los principales impulsores de esta realidad: en primer lugar, las posibilidades que hoy en día nos brindan las tecnologías de la información y la comunicación (TICs). En segundo lugar, la propia complejidad de nuestra sociedad, que exige un aprendizaje permanente ante la celeridad de los cambios económicos, tecnológicos, científicos, entre otros, que está sufriendo. En tercer lugar, la búsqueda de nuevos mercados ante la crisis económica que está afectando a todas las organizaciones, no quedando las educativas ajenas a este problema. En cuarto lugar, la irrupción de nuevos escenarios de aprendizaje, como resultado de las situaciones anteriores, lo que multiplica el surgimiento de propuestas telemáticas novedosas y un enriquecimiento de sus posibilidades de formación (García Aretio, 2007). El hecho más importante y que marca en la actualidad a esta modalidad educativa, ha sido la transformación tecnológica y su aplicación al campo de la educación.

La llegada de internet y sus múltiples herramientas, como el correo electrónico, listas de distribución, chat, blogs, news, wikis, entre otras, dan un nuevo significado y poder a la educación a distancia, generando la posibilidad de enseñar y aprender a través de la red. Con los avances tecnológicos, en los últimos tiempos se facilitó la comunicación bidireccional, que genera una retroalimentación mucho más fluida y rica y, sobre todo, favorece la interacción entre profesores y alumnos y entre los propios estudiantes entre sí, lo que supuso nuevas propuestas para la educación a distancia, rompiéndose la linealidad y la formación magistral tradicional en todo proceso de enseñanzaaprendizaje.

Igualmente, la educación a distancia ha pasado de ser considerada una enseñanza meramente instructiva, de dudosa validez y eficacia en la que prácticamente era imposible establecer una interacción educativa, a ser una modalidad de presente y de indudable futuro, dada la comunicación e interacción mediada por soportes tecnológicos que es capaz de generar, independientemente de los condicionamientos de espacio y tiempo que puedan presentarse. Sin duda, el elemento que más ha favorecido esta interactividad y que ha impulsado la educación a distancia a la conquista de estos nuevos escenarios han sido precisamente las tecnologías de la información y la comunicación, conocidas como TICs, (García Aretio, 2004).

Para Vázquez (2007), la educación a distancia amerita, por un lado, acciones de autoprogramación, autonomía, responsabilidad $y$ autocontrol por parte del estudiante $y$, por otro, requiere una estructura en términos de contenido y actividades, plataforma tecnológica de fácil acceso y manejo por parte de los usuarios, recursos y materiales diseñados para tal fin, así como un papel docente, que vaya más allá de transmitir información y que el alumno comprenda los contenidos.

\section{Características de la educación a distancia}

Las características que propone García Aretio (2006) sobre la educación a distancia, es que existe una permanente separación del profesor-formador, y alumno-participante en el espacio y tiempo; característica presente explícita o implícitamente en cualquiera de las diferentes modalidades en que se puede dar en esta modalidad, existe una separación geográfica entre los actores, pero que admite comunicación síncrona de recursos como teléfono, chat, videoconferencia, entre otros.

La educación a distancia supone un aprendizaje individual, privado y no grupal, preferentemente, pero siempre hay una institución detrás, cuya finalidad es apoyar al estudiante, motivarlo, guiarlo, facilitarle y evaluar su aprendizaje. En estamodalidad se considera a la asesoría presencial-grupal, un instrumento valioso para fomentar la socialización, la interacción y, aprovechar las oportunidades didácticas que ofrece el sistema, estas no deben ser excesivas ni representar la mayoría de las interacciones.

Aquí el estudiante toma decisiones con respecto a su propio proceso de aprendizaje, es una independencia motivada como resultado de la separación espacial del profesor. Así mismo la flexibilidad es clave de la independencia, que permite al estudiante elegir el tiempo, forma, espacio y ritmo, se trata de un control voluntario del proceso de aprender.

\section{Ventajas de la educación a distancia}

Según Restrepo (2000), algunas de las ventajas que ofrece la educación a distancia aparecen las siguientes: Hay variedad en el método de aprendizaje, se facilita el tratamiento, presentación y comprensión de cierto 
tipo de información, facilita que el alumno se vuelva protagonista de su propio aprendizaje, se optimiza el trabajo, motivación para el trabajo colaborativo, apertura de la clase a situaciones fuera del alcance del alumno, evita la movilidad de personas hacia el lugar del curso, ahorro de recursos, como hospedaje, alimentación, transporte.

\section{Desventajas de la educación a distancia}

Algunas de las desventajas que se pueden mencionar en la educación a distancia, aparecen, las dificultades para el proceso de socialización, poco espacio para el trabajo de interacción personal de los alumnos y profesores, existe poca o nula interacción en el ámbito afectivo y actitudinal de los alumnos entre pares y con sus profesores, así como personal administrativo, lo cual puede generar en los alumnos altos grados de ansiedad, sentimientos de soledad, abandono, falta de pertenencia a un grupo, Lozano (2007).

La retroalimentación puede ser muy lenta, la procedencia de estudiantes de instituciones tradicionales, la relación de dependiente profesor-alumno no se da bajo esta modalidad, lo cual puede repercutir en la eficacia de los sistema de enseñanza para lograr aprendizajes reales, la falta de disponibilidad de internet a un amplio sector de la población, altas deserciones, el gasto que representa la inversión inicial tanto para la institución como para el alumno, y escasez de personal directivo competente y conocedores del sistema.

La educación a distancia ha transitado por diferentes modalidades (García, Aretio, 2007) el e-learning, que corresponde a aquel aprendizaje que se produce por un medio tecnológico-digital; el m-learning, que es un modelo de enseñanza a distancia cuya base radica en el uso de servicios móviles o inalámbricos y el B-learning, que es un aprendizaje mezclado o hibrido, en donde se combina experiencias de aprendizaje presencial y a distancia.

\section{Metodología e-learning}

El término e-learning. Según Ruipérez (2003), se trata de una enseñanza a distancia caracterizada por una separación física entre el profesor y el alumno, donde entre cada uno de ellos predomina una comunicación de doble vía asíncrona, la cual utiliza el internet como medio de comunicación.

En concordancia con esto Mínguez (2003), coincide en que el e-learning es un aprendizaje basado en tecnología, que incluye la creación de contenidos, publicación, envíos, la administración del proceso de aprendizaje, evaluación, y retroalimentación. Como proceso de aprendizaje, el e-learningse apoya en las TICs. El elearning posibilita que cada estudiante pueda recibir los contenidos adaptados a sus estilos de aprendizajes predominantes, en donde frecuentemente, los materiales para la educación a distancia, se diseñan sin haber considerado la heterogeneidad grupal.
El trabajo en línea o bien el e-learning, a diferencia de la modalidad presencial, requiere que el profesor posea ciertos tipos de competencias y desarrollo de las mismas. Sin embargo, es importante darnos cuenta que el docente recurre a diferentes modalidades con el fin de ser creativo y aportar nuevas técnicas a sus clases.

Borges (2005) considera que tomar un curso elearning, implica saber manejarse en el entorno virtual, saber en dónde está lo que uno necesita y cómo conseguirlo, leer y escribir mensajes, leer y estudiar el material de aprendizaje, rendir actividades y ejercicios, y finalmente superar pruebas de evaluación.

A pesar del tiempo en que se lleva aplicando esta nueva metodología de aprendizaje, y los grandes beneficios y ventajas que conlleva, se escuchan las críticas de insatisfacción en la formación en línea. En este sentido Seoane (2005), afirma que algunas de las causas de la desmotivación, está el entorno de aprendizaje, los diseños de las infraestructuras, lo logístico, la cultura tecnológica, y la desmotivación por ausencia del factor humano en la formación en línea.

La estrategia de definición de un aprendizaje elearning es igual de importante que su interpretación por parte de los participantes, dado que si el curso en línea está mal diseñado también lo estará su contenido y el aprendizaje que se obtenga de él. Por ello Mendoza (2003), menciona que la participación de expertos es necesaria para implementar un buen programa, para que de esta manera se optimice el aprestamiento en lasinstitucioneseducativas.

\section{Metodología Blended Learning}

La palabra Blended quiere decir mezclar, entrelazar, y el término Blended Learning denota estrategias que combinan o mezclan metodologías o formatos para lograr mejores resultados de aprendizaje, Mortera F. (2009). Blended Learning específicamente se usa para referirse a la combinación de educación presencial y en línea, y se puede definir como la integración de elementos comunes a la enseñanza presencial, con elementos de la educación a distancia por Internet (Andrade, 2007). Esta metodología se le puede asociar a un proceso deenseñanza-aprendizaje flexible.

El modelo Blended Learning es una modalidad educativa que trata de superar las desventajas o dificultades del e-learning e igualmente presentan elementos al interior que podrían estar dificultando la permanencia y egreso de los estudiantes en una institución educativa.

En el modelo B-learning el profesor ejerce su labor de dos formas: como tutor online (tutorías a distancia) y como educador tradicional (cursos presenciales). La forma en que combine ambas modalidades dependerá de las necesidades específicas del curso, dotando así a la formación online de una gran flexibilidad (Gonzales, S. 2006) 
Para Oblinger (2001) esta modalidad de aprendizaje combinado, significa usar un amplio rango de tecnologías deinformación y comunicaciones para proveer oportunidades de aprendizaje más allá de las limitantes del aula de clase tradicional. Esta metodología permite que el tutor, los estudiantes y el contenido estén localizados en lugares diferentes, de forma descentralizada, de manera que la instrucción y el aprendizaje ocurren independientes de tiempo y de lugar (Gonzales S, 2006).

El aprendizaje combinado es particularmente eficaz en su capacidad de facilitar a una comunidad el dialogo y la capacidad de investigación. Por un lado la comunidad proporciona estabilidad y una comunicación abierta, que podrá ser contrastada o discutida por el acceso ilimitado a la información a través de internet, agregando un elemento importante que es el aprendizaje en grupo.

La pregunta clave en el BlendedLearning es respecto al necesario grado de socialización y alfabetización que deberían poseer los estudiantes para mantener una interacción provechosa, según los códigos simbólicos en los que aparecen los contenidos, ya que la selección de dichos recursos presenciales y no presenciales, depende de la disponibilidad existente y sus funcionalidades, que debe ser protagonizada por el estudiante a diferencia de lo que era establecido por el profesor (Fainhloc, 2006). El centro del proceso de enseñanzaaprendizajes esta en el estudiante, y no en el docente, como ocurre en el modelo tradicional.

Para Turpo O. (2009), la metodología Blended Learning es aquel modo de aprender que combina la enseñanza presencial con la tecnología no presencial. Por su parte García L (2009), no ven en la metodología Blended Learning, nada innovador, es más bien una metodología que se ha usado por años, lo que sí es novedoso según Mortera (2009) es el soporte científico de este modelo.

Agrega Turpo O. (2009), que anteriormente la enseñanza tradicional, la que se da cara a cara se apoyaba en elementos tecnológicos, mientras que hoy en día después de un auge de e-learning y el surgimiento de problemas en el proceso, se está apoyando la educación a distancia con presencialidad. Representa un modelo más amplio que incluye la utilización de escenarios formativos múltiples de acuerdo con la necesidad del que aprende.

Para autores como Fainhloc (2006), plantea que no es un modelo de aprendizaje basado en una teoría general del aprendizaje, sino la aplicación, al aprendizaje y a la enseñanza de líneas eclécticas y prácticas, usando las TICs, dentro de un marco que combina los recursos tecnológicos existentes, en donde se reducen los costos, y se mejora la enseñanza.

Mortera (2007), ha identificado que el BlendedLearning ha crecido en popularidad, como la combinación de la enseñanza tradicional y el e-learning. Singh \& Reed
(2001) definen BlendedLearning como una experiencia de aprendizaje que combina las formas offline y online de aprendizaje, donde online significa a través del internet y offline tiene lugar en un salón de clases tradicional.

Estos autores presentan estudios en el que sugieren, según las características de los participantes y el entorno de aprendizaje, un conjunto de pautas que permitan lograr la mezcla adecuada de elementos en línea y cara a cara, obteniendo como resultado el programa más efectivo para BlendedLearning. Se reconoce entonces que uno de los mayores retos es encontrar el verdadero balance en la integración de diversos elementos dentro de un mismo plan de formación.

Según Salinas (1999) y otros contemporáneos, consideran que el aprendizaje combinado o mixto apunta a un modo de aprender en el cual se combina una modalidad de enseñanza y aprendizaje presencial con una modalidad de enseñanza y aprendizaje virtual.

Esta modelo no específica que debe ir primero, pero en todo caso, se combina el rol tradicional de la clase presencial con el nuevo rol del tutor de educación a distancia. Más aún, se podría considerar que esta modalidad mixta parece ser una respuesta válida para mejorar la calidad de la educación porque reúne todas las características básicas de un proceso de enseñanza-aprendizaje, como lo afirman Forés \& Trinidad (2003), y Vera F. (2008). En este modelo el tutor asume un rol esencialmente de mediador del proceso de enseñanza y aprendizaje.

\section{Elementos de la metodología BlendedLearning}

De acuerdo a Driscoll \& Vergara (1997, citados en Zañartu, 2003), en la metodología B-learning, se consideran los siguientes elementos:

- Debe haber una responsabilidad individual, en todos los miembros que deben ser responsables de su desempeño individual dentro del grupo.

- Se presenta una Interdependencia positiva entre cada miembro del grupo para lograr una meta común.

- Las Habilidades de colaboración, deben ser necesarias para que el grupo funcione en forma efectiva, como el trabajo en equipo, liderazgo y solución de conflictos.

- Se debe tener presente una buena Interacción promotora, para que cada miembro del grupo interactúe, hacia un desarrollo de relaciones interpersonales, y se puedan establecer estrategias efectivas de aprendizaje.

- Trabajo grupal, que permita una reflexión permanente hacia una autoevaluación de su funcionamiento, desarrollando los cambios necesarios para incrementar la efectividad. 


\section{Modelos de BlendedLearning}

Vera F. (2008), afirma que esta metodología de enseñanza-aprendizaje, ofrece diversos modelos, los que combinan varias opciones, como clases en el aula física, clases en aula virtual, aprendizaje al propio ritmo, y aprendizaje colaborativo. Igualmente estos modelos de enseñanza BlendedLearning se clasifican según el uso de recursos TICs comparado con la enseñanza presencial, o según el nivel de integración de Internet en la clase presencial (Fainholc, 2006). Algunos de los modelos más conocidos bajo esta modalidad de formación son:

\section{Modelo GI(GroupInvestigation)}

El modelo GI se caracteriza por ser pragmático, por cuanto involucra investigación, interacción, interpretación, y motivación intrínseca. Este modelo permite la conformación de grupos heterogéneos de cinco a seis miembros. Para su trabajo se selecciona un tema de estudio, luego el equipo plantea la estrategia de aprendizaje que utilizará. La función del tutor es supervisar y apoyar el trabajo.

\section{Modelo JIGSAW}

Este modelo mixto permite la conformación de grupos heterogéneos con un bajo número de integrantes (entre cinco y seis miembros). El material de trabajo debe ser distribuido entre cada miembro, para luego ser socializado de manera integral. Por lo general, en este modelo se utiliza un asesor tutor que acompañe el proceso de aprendizaje.

\section{Modelo STAD (Student Teams Achievement Divisions)}

El modelo STAD, presenta como característica el agrupamiento heterogéneo de individuos, en donde cada individuo tiene la responsabilidad de apoyar a sus compañeros, con explicaciones, debates y/o ejercicios, aunque las evaluaciones son realizadas de manera individual. Lo que se busca es mejorar el desempeño del grupo.

En general estos modelos de aprendizaje, son eficaces por cuanto facilita a una comunidad académica el dialogo y la capacidad de investigación. Por un lado la comunidad proporciona estabilidad y una comunicación abierta que podrá ser contrastada o discutida por el acceso ilimitado a la información a través de las TICs, agregando un elemento importante que es el aprendizaje colaborativo grupal.

\section{Conclusiones}

Los modelos Learning apoyados enTICs representan uno de los principales factores de cambio de las instituciones de educación superior, pues, según el Informe de Bricall (2000), estas auguran en el campo educativo la progresiva desaparición de las restricciones de espacio y de tiempo en la enseñanza y la adopción de un modelo de aprendizaje más centrado en el estudiante. Al mismo tiempo, favorecen la comercialización y la globalización de la educación superior, así como un nuevo modelo de gestión de su organización. (Vera, F. 2008).

Para diseñar un curso en educación a distancia, no se debe perder de vista lo esencial, que es el learning, pues éste es el elemento que producirá en los alumnos un aprendizaje y les dará las herramientas necesarias para enfrentarse al mundo (Lozano R, 2007).

El proceso de enseñanza-aprendizaje B-learning permite tomar y aprovechar los mejores elementos tanto de las experiencias presenciales, como de las de educación a distancia, enriqueciendo aún más el aprendizaje de los alumnos y su desarrollo de habilidades. Al tener contacto cara a cara con maestros y alumnos, se pueden evitar los sentimientos de soledad que éstos puedan sentir y al tener la experiencia y enriquecimiento con actividades a distancia pueden obtener todos los beneficios que esta modalidad educativa trae a su aprendizaje y desarrollo.

B-Learning, se centra en la combinación de estrategias pedagógicas, inmersas en los modelos presenciales, y estrategias formativas propios de e-learning, por lo que no debemos desconocer, ni dejar de lado estas enriquecedoras metodologías que se apoyan en recursos educativos abiertos, que irá a enriquecer el proceso de enseñanza-aprendizaje.

\section{Referencias Bibliográficas}

Andrade, A. (2007) Aprendizaje combinado como propuesta en la convergencia europea para la enseñanza de las ciencias naturales. E-learningpapers. No 3 Recuperado el 16 de agosto de 2011 en: www.redeees.com/.../articulo-elearning-papers-aprendizajecombinado.

Borges, F. (2005). La Frustración del estudiante en línea. Causas y acciones preventivas. Digithum, (UCO No.7) 1-8.

Bricall J. (2000) Conferencia de rectores de universidades españolas. Informe Universidad 2000. Recuperado el 20 de abril de 2011 de http://www.oei.es/oeivirt/bricall.htm

Fainholc B (2006) Optimizando las posibilidades de las TICS en educación. Revista electrónica de tecnología educativa.Num 22. Recuperado el 24 de abril de 2011 de http://edutec.rediris.es /Revelec2/revelec22/beatriz.pdf

Forés A., Trinidad C. (2003) Amalgama o puzle?El blended learning. Educaweb.Nro. 69. ISSN 1578-5793. Recuperado el 7 de febrero de 2011 de:www.educaweb.com/esp/monograficos

García Aretio, L. (2006). La educación a distancia: De la teoría a la práctica. Barcelona, España: Ariel Educación.

García Aretio, L. (2007). De la Educación a Distancia a la Educación Virtual. Barcelona España: Ariel Educación.

García, L.(2004). Blended Learning, ¿es tan innovador?Editorial del BENED.

Gonzales, S. (2006) Un modelo Blendedlearning para la enseñanza de la educación superior. Virtual educa 2006. Recuperado el 26 marzo 2011, en: http://ihm.ccadet.unam.mx/virtualeduca2006 /pdf/133-SGS.pdf

Gil, M. (2000) Reseña de educación a distancia de la teoría a la práctica. Perfiles educativos. UNAM Num 88

Jiminián, Y. (2006) Causas de la deserción en la educación a distancia. El caso de la UAPA. Revista de educación Superior. Vol V No 1 y 2. Pp 7-18.

Lozano, A. (2007). Reconocimiento de los estilos de aprendizaje en cursos ofrecidos en línea. En Lozano Rodríguez, A. y Burgos Aguilar V. (Comp.) Tecnología educativa en un modelo de educación a distancia centrado en la persona. Distrito Federal, México: Limusa 
Mendoza, J. (2003). E-learning, el futuro de la educación a distancia. Recuperado el 9 de octubre de 2011 de: http://www. informaticamilenium.com.mx/paginas/mn/articulo78.htm

Mínguez, A. (2003). El formador en la empresa. Madrid: Esic Editorial

Mortera F. (2009) Diferencia y similitudes entre el aprendizaje combinado (BlendedLearning y el aprendizaje distribuido (distributedlearning), y su relación con la educación a distancia. Revista Escuela Normal "Miguel F Martínez" centenaria y benemérita. Año 3 Num 4. Recuperado el 22 de abril de 2011 de http://prug.espacios.net.mx/wccenmfm/sitio/archivos/3_w_revi sta_EN4.pdf

Mortera, F. (2007). El aprendizaje hibrido o combinado

(BlendedLearning): acompañamiento tecnológico en las aulas del siglo XXI. En Lozano Rodríguez, A. y Burgos Aguilar V. (Comp.) Tecnología educativa en un modelo de educación a distancia centrado en la persona. Distrito Federal, México: Limusa.

Oblinger D, Maruyama M (2001). Distributed learning. CAUSE professional Paper Series, 14. Boulder. Recuperado el 9 de Julio de 2011 de: Http://eric.ed.gov/ERICWebportal/search/detailmini. jsp? nfpb=tru\&_\&ERICExtsearch_searchValue_0=ED396647\&\&RI CExtSearch_SearchType_0=no\&accno=ED396647

Restrepo M (2000) et al. Conexiones, Informática y Escuela: Un enfoque global. Medellín: Editorial Universidad Pontificia Bolivariana; 2000:48.

Ruipérez, G. (2003). Educación virtual y eLearning. Madrid: FundaciónAuna.

Salinas J. (1999).Enseñanza flexible, aprendizaje abierto. Las redes como herramientas para la formación. Revista Electrónica de Tecnología Educativa. Num 10. Recuperado el 8 de Junio de 2011 en: http://www.uib.es/depart/gte/revelec10.html

Seoane A (2005) Causas de la insatisfacción en la formación on-line Algunas ideas para reflexionar. Educaweb.com. Recuperado el 8 de marzo de http://www.educaweb.com/noticia/2005/09/26/ causas-insatisfaccion-formacion-on-line-algunas-ideas-reflexion1680.html

Serrano C, López L. (1999) Educación a distancia: su polisemia y su prospectiva en la educación pública. Tiempo de educar.Vol 1;2pp 71-92.

Sing H, Reed C (2001). A white paper: Achieving success with Blended Learning.

Turpo 0. (2009) Desarrollo y perspectiva de la modalidad educativa blendedlearning en las universidades de Iberoamérica. Revista Iberoamericana de Educación. Vol 50 num 6 2009. Recuperado el 16 de abril de 2011 en http://www.rieoei.org/deloslectores/3002 Gebera.pdf

Vázquez C. (2007) La deserción estudiantil en educación superior a distancia: perspectiva teórica y factores de incidencia. Revista Latinoamericana de Estudios Educativos. Vol XXXVI, No 3-4, pp 107-122

Vera, F. (2008). La modalidad Blended-Learning en la educación superior. Recuperado 21 de abril de 2011 , de http://www.utemvirtual.cl/nodoeducativo/wpcontent/uploads/ 2009/03/fvera_2.pdf

Zañartu, L. M. (2003). Aprendizaje colaborativo: Una nueva forma de diálogointerpersonal y en red. Revista Digital de Educación y Nuevas Tecnologías. Recuperado el 19 de Septiembre de 2011 de: http://contextoeducativo.com.ar/2003/4/nota-02.htm 